\title{
Experimental validation of a CFD-based resistive force coefficient set for rotating helical tails in cylindrical channels
}

\author{
A. Fatih Tabak $^{1} \&$ S. Yesilyurt ${ }^{2}$ \\ ${ }^{1}$ Faculty of Engineering and Design, \\ Istanbul Commerce University, Turkey \\ ${ }^{2}$ Faculty of Engineering and Natural Sciences, \\ Sabanci University, Turkey
}

\begin{abstract}
Real-time manipulation of objects in micro flows is important due to an ever increasing interest in demanding medical applications. Specialized swimming micro robots are expected to perform minimal invasive surgery consisting of various in vivo tasks. Moreover, fluid forces exerted on moving surfaces are crucial for maneuverability if swimming micro robotic tools are ever to play a key role in therapeutic applications. Hydrodynamic forces acting on an isolated object of regular blunt shapes immersed in micro flows are calculated by resistive force coefficients based on the Stokes Flow approximation derived by analytical means. However, force coefficients presented in literature often lack the accuracy to predict the hydrodynamic interactions between rotating and translating objects of irregular shapes, and rigid concave surfaces such as a rigid helix moving inside a cylindrical channel. In this study a set of parameterized 3D CFD simulations are carried out using a novel geometric representation for rigid helical tails of varying sizes and of varying distances with respect to the wall of a cylindrical channel. Furthermore, the complex rigid-body motion of the helix is represented by a series of simple translations and rotations. Each simulation is governed by Stokes equations and carried out by a commercial CFD package. A generic resistive force coefficient set is obtained via surface-fit procedures based on the hydrodynamic forces computed by the CFD simulations along the tangential, normal and binormal directions on the moving and rotating helical surface. Finally, using a reduced-order microhydrodynamic model, the proposed coefficient set is validated with empirical data collated using bio-inspired cm-
\end{abstract}


scale robots autonomously swimming in a cylindrical channel filled with silicone oil of high viscosity. The robots are on-board powered and propel themselves with rotating rigid helices of parameterized geometric features. The CFD-based coefficient set predicted the experiment-based velocities of the bio-inspired untethered robots with good agreement.

Keywords: cylindrical channels, bio-inspired robots, untethered swimming, low Reynolds number flows, CFD analysis, resistive force coefficients, reduced-order models.

\section{Introduction}

Microswimmer robots are expected to be highly beneficial in medical studies in the near future with possible applications of minimal invasive surgery such as kidney stone destruction, retina repair, opening clogged arteries [1-3]. Controlled swimming of bio-inspired robots is crucial to high precision in-vivo and in-vitro applications [4-6].

Kinematic and dynamic modeling of bio-inspired swimmer robots rely upon resistive force coefficients to predict the hydrodynamic thrust generated by the articulated tail carrying out helical or planar wave propagation and the net viscous drag acting on the payload against the swimming direction [7]. However, these coefficient sets are mostly applicable for slender tails actuated in unbounded mediums of high viscosity [8] with a few exceptions for slender bodies moving near solid plane walls $[9,10]$.

Furthermore, there exist approximate analytical solutions based on harmonic functions or Stokeslet functions representing the induced flow field due to the helical wave propagation in viscous fluids confined to cylindrical channels $[11,12]$.

In this study, a novel coefficient set is implemented via CFD simulations and validated with experimental results. Untethered bio-inspired robots with rigid rotating helical tail are built and employed to study the dependence of swimming behavior on parameterized helical tail geometry, i.e. wave length and wave amplitude. A reduced order model based on resistive force approach is implemented to predict the observed swimming velocities. In order to obtain a generic coefficient set to predict the net thrust generated by rotating helical tails, a CFD study is carried out on pairs of a separate torus and a separate rod, which together represent a full helical wave of different geometric configurations. Local fluid forces exerted on separate torus and rod geometries induced by their relative motion to the cylindrical walls are carefully examined under parameterized wall proximity, tail radius and wave amplitude. Computations are used to implement the resistive force coefficient sets for the torus and the rod individually via surface fitting. Finally these coefficient sets are incorporated into an effective force coefficient set for a complete helical wave in the reduced order model. Performance of the new set is compared against another coefficient set which is presented for swimming near plane walls in the literature. 


\section{Methodology}

\subsection{Experimental Setup}

Figure 1 demonstrates the components of the cm-scale robotic prototype. Payload of the untethered robot is designed as a sealed case comprised of a silica glass cover and a plastic cap, which holds a rechargeable Li-Po battery, a coreless brushed DC-motor, an IR-receiver and PWM controller circuit. The mechanical power is transmitted to the rigid helical tail by a coupling element. Rigid helical tails are made out of copper wire of $1 \mathrm{~mm}$ diameter by winding and deforming to desired wave geometries and are actuated via the dedicated DCmotor. The bullet shaped payload is $18 \mathrm{~mm}$ in diameter and all helical tails presented in this study are of the same apparent length of $60 \mathrm{~mm}$. Manufacturing details are discussed elsewhere in detail [13].

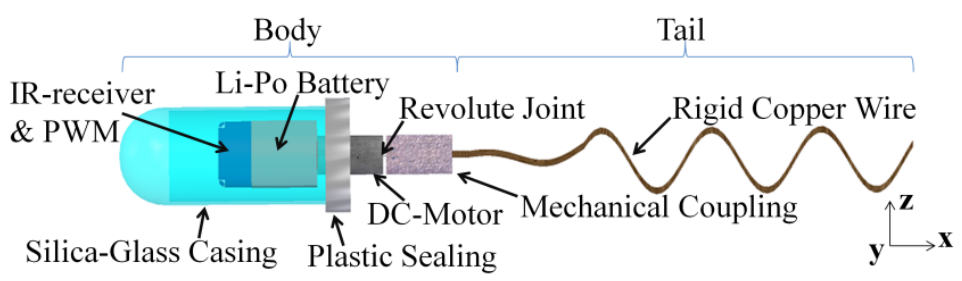

Figure 1: Bio-inspired robotic prototype.

Figure 2 illustrates the experimental setup for the in-channel swimming studies. The untethered robot is placed in a glass channel of $30 \mathrm{~mm}$ in diameter and actuated with an IR-controller. The channel is filled with silicone oil of 3.5 Pa`s viscosity and both ends of the channel are sealed forming a confined medium. The channel is placed in a silicone oil bath horizontally and a $30 \mathrm{fps}$ CCD-camera is placed $15 \mathrm{~cm}$ above to capture the swimming action. The details of the experimental procedure and helical tail actuation are provided elsewhere $[14,15]$. Important details of the experimental setup are presented in Table 1.

Table 1: Important properties of experimental setup.

\begin{tabular}{|l|l|}
\hline Channel Diameter & $30 \mathrm{~mm}$ \\
\hline Channel Length & $350 \mathrm{~mm}$ \\
\hline Total Swimmer Length & $105 \mathrm{~mm}$ \\
\hline Body Diameter & $18 \mathrm{~mm}$ \\
\hline Helical Tail Length & $60 \mathrm{~mm}$ \\
\hline Copper Wire Diameter & $1 \mathrm{~mm}$ \\
\hline Helical Wave Amplitude & Parameterized \\
\hline Helical Wave Length & Parameterized \\
\hline Si-Oil Density & $985 \mathrm{~kg} / \mathrm{m}^{3}$ \\
\hline Si-Oil Viscosity & $3.5 \mathrm{~Pa} \cdot \mathrm{s}$ \\
\hline CCD-Camera & $640-\mathrm{by}-480$ Pixels @ $30 \mathrm{fps}$ \\
\hline CCD-Camera Elevation & $150 \mathrm{~mm}$ \\
\hline Li-Po Pack Rating & $70 \mathrm{~mA} \cdot \mathrm{h}$ with $3.7 \mathrm{~V}$ \\
\hline
\end{tabular}



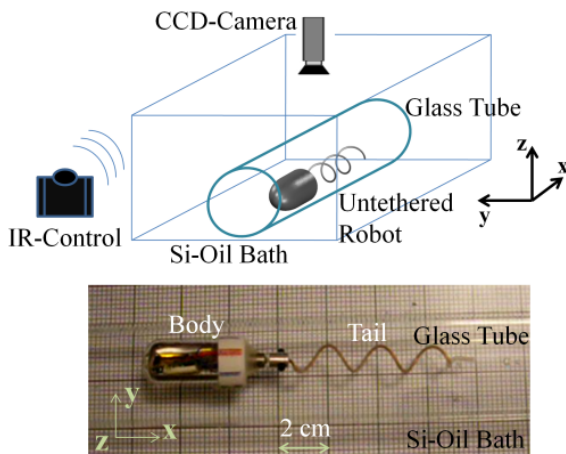

Figure 2: $\quad$ Experimental setup and the swimmer inside the silica glass tube.

\subsection{Reduced-order microhydrodynamic model}

The model implemented in this study is based on the resistive force theory where all inertial forces are neglected due to the fact that the dynamic behaviour of the robot is primarily dominated by the hydrodynamic shear acting on its surfaces $[16,17]$. In this scheme, the fluid forces acting on a surface can be expressed in a linear relationship with the local velocity of that surface [18].

The equation of motion in order to find the swimming velocity of the untethered robot is given by [15]:

$$
\mathcal{F}_{\mathrm{b}}+\mathcal{F}_{\mathrm{t}}+\mathcal{F}_{\text {eff }}=0
$$

where $\mathcal{F}$ is the six-by-one generalized resistance force vector acting on robot surfaces, subscripts b, t and eff denote the surfaces of body, surfaces of the tail and effective rotational friction torque acting on the robot respectively. The hydrodynamic force acting on the body and tail of the robot are specified as:

$$
\mathcal{F}_{\mathrm{b}, \mathrm{t}}=-\mathbf{B}_{\mathrm{b}, \mathrm{t}} \mathbf{U}_{\mathrm{b}, \mathrm{t}}
$$

where, $\mathbf{U}$ is the generalized six-by-one rigid body velocity vector, and $\mathbf{B}$ is the corresponding six-by-six fluid resistance matrix.

The generalized velocity vector of the body is also the swimming velocity of the robot. Furthermore, the generalized velocity vector of the helical tail includes the actuation, i.e. rotation with respect to its long axis. Given that the local position of an arbitrary point on the tail with respect to center of mass is given by vector $\mathbf{P}$, the rotational velocity vector of a right-handed helix with respect to robot's center of mass is obtained from its time derivative as $\mathrm{d} \mathbf{P} / \mathrm{d} t=\mathbf{\Omega}_{\mathrm{t}} \times \mathbf{P}$ where the tail rotation rate is $\boldsymbol{\Omega}_{\mathrm{t}}=\left[\begin{array}{lll}\omega & 0 & 0\end{array}\right]^{\prime}$.

The resistance matrix $\mathbf{B}_{\mathrm{b}}$ for the rigid body is implemented similar to $\mathbf{B}_{\mathrm{t}}$; however, effortlessly based on the fact that the body of the swimming robot is a blunt object [19]. Furthermore, the effective rotational friction $\mathcal{F}_{\text {eff }}$ acting on the robot is treated separately than standard fluid resistance forces and calculated 
with the help of actuation system dynamics [15] which is beyond the scope of this work.

Tail resistance matrix $\mathbf{B}_{\mathrm{t}}$ is obtained from the integration of the local force coefficients which are projected onto the lab coordinates as:

$$
\mathbf{B}_{\mathrm{t}}=\int_{\mathrm{L}_{\mathrm{t}}}\left[\begin{array}{cc}
\mathbf{R} \mathbf{C} \mathbf{R}^{\prime} & -\mathbf{R} \mathbf{C} \mathbf{R}^{\prime} \mathbf{S} \\
\mathbf{S} \mathbf{R} \mathbf{C} \mathbf{R}^{\prime} & -\mathbf{S} \mathbf{R} \mathbf{C} \mathbf{R}^{\prime} \mathbf{S}
\end{array}\right]\left(\ell / \mathrm{L}_{\mathrm{t}}\right) \mathrm{d} x
$$

where $\ell$ is the actual length of the wire, $\mathbf{S}$ is the skew-symmetric matrix corresponding to the cross product with local the position vector on the tail, $\mathbf{R}$ is the rotation matrix between the local Frenet-Serret coordinates, i.e. tnb-frame, and the robots' own frame of reference [20].

The local resistance on the tail, denoted by $\mathbf{C}$ in resistance matrix of the tail, is considered as a diagonal matrix that consists of the elements of local resistance coefficient set explicitly articulated for local in the tangent, binormal, and normal directions [15].

There are several approximations for the viscous drag exerted on slender objects submerged in unbounded mediums [7, 8, 21] and moving near solid boundaries [9-12, 22, 23]; however, none presents a resistive coefficient set for helical tails moving near cylindrical walls. Given the linearity of the Stokes Flow approximation, it is plausible to represent a complex geometry such as a finite helix as the combination of simpler blunt objects, e.g. a series of slender tori and rods. The effective $\mathbf{C}$ matrix then could be implemented as:

$$
\mathbf{C}=\mathbf{C}_{\text {torus }}+\mathbf{C}_{\text {rod }}
$$

with superimposed drag coefficients in tnb-frame based on local projection due to the attack angle $\zeta$ (see Figure 3 ) of helical tails [15]:

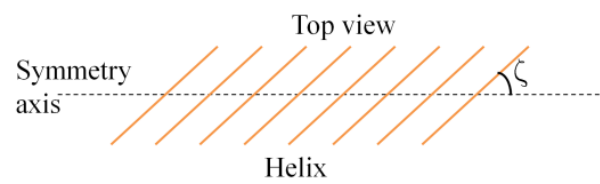

Figure 3: $\quad$ Attack angle $\zeta$ of the helical tail.

$$
\begin{aligned}
& c_{t}=c_{t}^{\text {rod }} \cos (\zeta)+c_{t}^{\text {torus }} \sin (\zeta) \\
& c_{n}=c_{n}^{\text {rod }} \cos (\zeta)+c_{n}^{\text {torus }} \sin (\zeta) . \\
& c_{b}=c_{b}^{\text {rod }} \cos (\zeta)+c_{b}^{\text {torus }} \sin (\zeta)
\end{aligned}
$$

and the local force coefficients are formulated as:

$$
c_{\{t, n, b\}}^{\{\text {rod,torus }\}}=\frac{\pi \mu}{\left[a \ln \left(\frac{\mathrm{d}}{r_{\text {tail }}}\right)+b \ln \left(\frac{R_{c h}}{r_{\text {tail }}}\right)+c\left(\frac{\mathrm{d}}{R_{\text {ch }}}\right)^{d}+e\right]}
$$


where constants $\{a, b, c, d, e\}$ are to be determined by surface fit analysis, $\mu$ is the dynamic viscosity, $\mathrm{d}$ is the local proximity to the channel walls, $R_{c h}$ is the channel radius, and $r_{\text {tail }}$ is the radius of the helical tail. This new set incorporates the corrections for the ratios of $\mathrm{d} / r_{\text {tail }}, R_{c h} / r_{\text {tail }}$ and $\mathrm{d} / R_{c h}$ together. Furthermore, the local proximity, i.e. $d$ in the above equation, is not the shortest local distance between but the shortest local distance within the same quadrant of helical tail and channel. It also is noted that the equation postulated here is determined by several trial-error steps on the solution of the surface fit study.

The next section presents a detailed discussion on how to conduct numerical experiments to predict fluid resistance vector exerted on a torus and rod undergoing certain rigid-body motions near solid boundaries.

\subsection{CFD simulations and surface fit}

The CFD study based on a design space sweep for dimensions and proximity of well known geometries followed by a surface fit provides a novel set of force coefficients. In the following study the helical tail is assumed to be concentric with cylindrical channel of unit radius.

Open boundary conditions are set at the channel inlet and outlet boundaries to analyze the torus. No-slip boundary condition is imposed on channel walls. Motion in tangential, normal, and binormal directions of the torus (see Figure 4) are studied separately, with unit velocity boundary conditions imposed on its surface as $\mathbf{U}_{\text {torus }}=\left[\begin{array}{lll}2 \pi & 1 & 1\end{array}\right]$ ', respectively. The fluid stress vector per unit length of the torus in tnb-frame is calculated by integrating the fluid stress [24] over entire surface area as:

$$
\begin{gathered}
\mathbf{F}_{\mathbf{n b}}^{\text {torus }}=\frac{1}{2 \pi R_{\text {torus }}} \int_{S_{\text {torus }}}[-p \mathbf{I}+\boldsymbol{\tau}]_{\mathbf{n b}} \mathrm{d} A, \\
\mathbf{F}_{\mathbf{t}}^{\text {torus }}=\frac{1}{2 \pi R_{\text {torus }}^{2}} \int_{S_{\text {torus }}}\left(\Re_{\text {torus }} \times[-p \mathbf{I}+\boldsymbol{\tau}]\right)_{\mathbf{t}} \mathrm{d} A
\end{gathered}
$$

where $R_{\text {torus }}$ is the major radius of the torus, $\Re_{\text {torus }}$ is the local position vector of an arbitrary point on the torus, $p$ is the static pressure, $\boldsymbol{\tau}$ is the viscous stress tensor, $A$ is the area, and $S_{\text {torus }}$ denotes the total surface area of the torus.

As opposed to the previous analysis, in order to simulate the rod in a channel, periodic boundary conditions [25] are set at the channel inlet and outlet boundaries. Hence, the rod intersects with the inlet and outlet perpendicularly to eliminate the end effects. Moreover, periodic boundary conditions imply that the studied rod is only a smaller segment of the actual geometry. Similarly to the torus study, no-slip boundary condition is imposed on channel walls. Motion in tangential, normal, and binormal directions of the rod (see Figure 5) are studied separately, with unit velocity boundary conditions imposed on its surface as $\mathbf{U}_{\text {rod }}=\left[\begin{array}{lll}1 & 1 & 1\end{array}\right]^{\prime}$, respectively. 


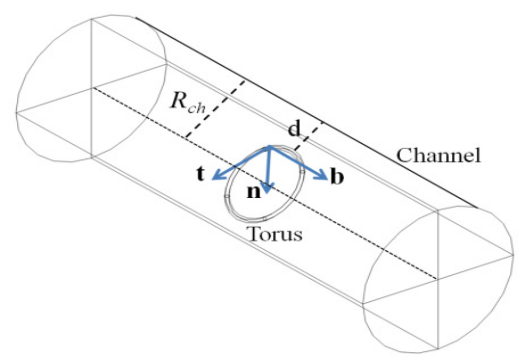

Figure 4: Concentric torus in the cylindrical channel. Tangential direction points the rigid-body rotation long the symmetry axis; binormal direction points the rigid-body translation along the symmetry axis; normal direction points rigid-body translation in radial direction.

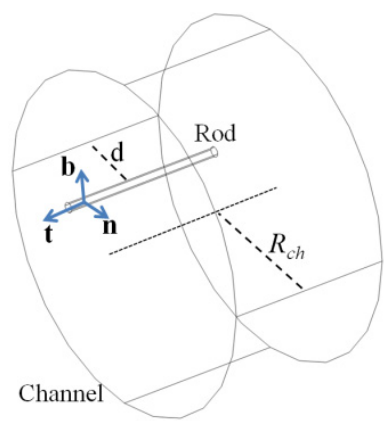

Figure 5: Rod in a channel with periodic boundary conditions. Tangential direction points the rigid-body translation along the symmetry axis; binormal direction denotes the instantaneous lateral translation due to rigid-body rotation; normal direction points rigid-body translation in radial direction.

The fluid stress vector acting on per unit length of the rod is calculated by:

$$
\mathbf{F}_{\text {tnb }}^{\text {rod }}=\frac{1}{\mathrm{~L}_{\text {rod }}} \int_{S_{\text {rod }}}[-p \mathbf{I}+\boldsymbol{\tau}]_{\text {tnb }} \mathrm{d} A
$$

where $\mathrm{L}_{\text {rod }}$ is the length of the rod, and $S_{\text {rod }}$ is the total surface area of the rod.

\section{Results}

CFD simulations are carried out with the fluid domain governed by steady incompressible Stokes equations subject to conservation of mass. Dynamic viscosity, $\mu$, and density, $\rho$, of the fluid is set to 1 in order to incorporate the effect of viscosity in the resistive force coefficient set. Second order Lagrangian tetrahedral elements are used. Solutions are obtained by PARDISO direct solver 
[26] and each simulation requires approximately 10 minutes on an Intel Core i7 system with $4 \mathrm{~GB}$ of RAM.

A total of 936 individual CFD simulations are carried out for 13 different $r_{\text {tail }}$ values between $\{0.0025,0.05\}$ and 12 different $d$ values between $\{0.067,0.8\}$ for rigid-body motions of the torus and the rod in all three directions as depicted in Figure 4 and Figure 5. Corresponding surface fits are obtained by least square method with 95\% confidence interval (see Figure 6 for the surface fit on total fluid resistance $\sigma_{\mathrm{n}}$ due to radial motion of the torus). Details of the simulations and surface fit study are presented elsewhere [15]. Predicted parameters, i.e. $\{a, b, c, d, e\}$ in Equation (6), for resistive force coefficients of the torus and the rod are:

$$
\begin{aligned}
& \left\{\begin{array}{lllll}
a & b & c & d & e
\end{array}\right\}_{\mathbf{t}}^{\text {rod }}=\left\{\begin{array}{lllll}
1 & -0.5135 & -3 & 0.3 & 3
\end{array}\right\} \\
& \left\{\begin{array}{lllllllll}
a & b & c & d & e
\end{array}\right\}_{\mathbf{n}}^{r o d}=\left\{\begin{array}{lllll}
0.15 & 0.062 & 0.75 & 0.08333 & -0.8
\end{array}\right\} \\
& \left\{\begin{array}{lllllllll}
a & b & c & d & e
\end{array}\right\}_{\mathbf{b}}^{\text {rod }}=\left\{\begin{array}{lllll}
0.6 & -0.335 & -3 & 0.2 & 2.75
\end{array}\right\} \\
& \left\{\begin{array}{lllll}
a & b & c & d & e
\end{array}\right\}_{\mathbf{t}}^{\text {torus }}=\left\{\begin{array}{lllll}
0.15 & 0.05 & 0.237 & 6 & 0.05
\end{array}\right\} \\
& \left\{\begin{array}{lllll}
a & b & c & d & e
\end{array}\right\}_{\mathbf{n}}^{\text {torus }}=\left\{\begin{array}{lllll}
0.0705 & 0.2 & 1.5 & 0.25 & -1.3
\end{array}\right\} \\
& \left\{\begin{array}{lllllllll}
a & b & c & d & e
\end{array}\right\}_{\mathbf{b}}^{\text {torus }}=\left\{\begin{array}{lllll}
0.2 & -0.027 & 0.5 & 0.2 & -0.2
\end{array}\right\}
\end{aligned}
$$

It is observed that the coefficients corresponding to normal and binormal directions demonstrate the asymmetry of the induced flow field due to the presence of the cylindrical walls as opposed to swimming in an unbounded viscous medium. These coefficients can also be compared against the known resistive force coefficients based on Stokes flow approach [9, 21].

In the experimental study, images captured by CCD-camera are inspected frame-by-frame in order to resolve the body rotation rate, $\Omega_{b o d y}$, and tail rotation rate, $\omega_{\text {tail }}$, along with swimmer's forward velocity $U_{\text {swimmer }}$. It is noted that lateral rigid body translations or rotations are observed to be insignificant and excluded. Furthermore, the condition of $\mathrm{Re}<0.1$ for Stokes flow approach to be valid is satisfied for each and every experiment.

There are sixteen rigid helical tails with four different amplitude and four different wave lengths. Each tail is used individually to conduct five distinct experiments; hence, velocity values are obtained by averaging out five distinct measurements for each quantity mentioned above.

The reduced-order hydrodynamic model is used to predict the forward velocity and body rotation rate of the swimmer while the tail rotation rates are used as inputs for each individual tail configuration. Time-dependent integrations are carried out by Adams-Bashforth-Moulton PECE solver [27] with a solution time of a few seconds and the time-averaged results presented here are obtained via averaging out two full periods.

Experimental results are predicted with both the CFD based novel coefficient set presented in this paper and the coefficient set given by Brennen and Winet [9] for slender rods moving near plane walls, which previously tested [14]. 


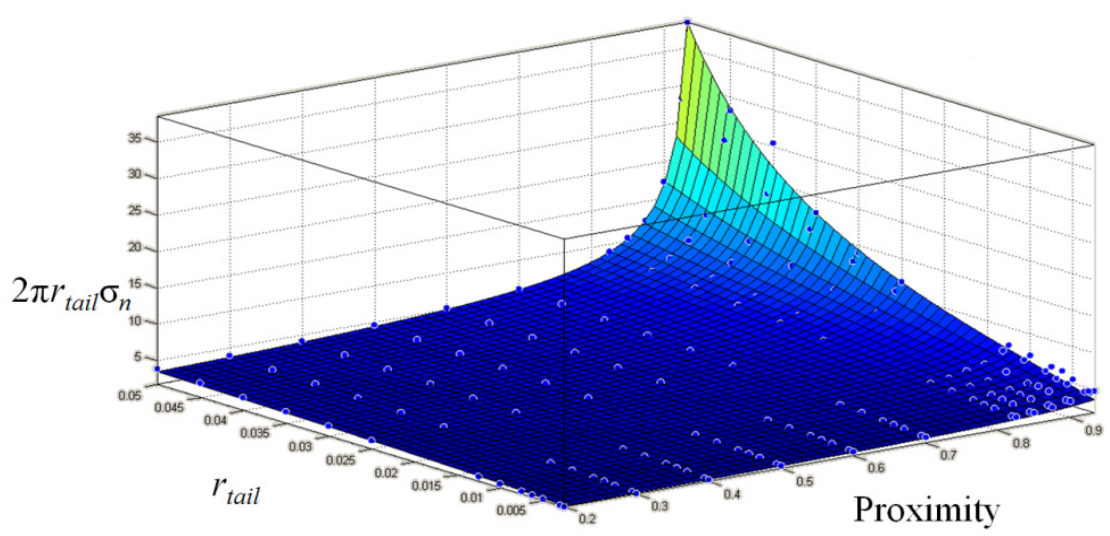

Figure 6: $\quad$ Surface fit for torus undergoing rigid-body translation in normal direction. (Adjusted R-square $=0.9998$, Root mean square error $=$ $0.06694)$.

Figure 7 demonstrates the performance of the coefficient sets against each other and the experimental results. In Figure $7, N_{\lambda}$ is the number of helical waves on the tail, $U_{\text {swimmer }} / U_{\text {wave }}$ is the ratio of the velocity of the swimmer to the velocity of the helical waves, $\Omega_{\text {body }} / \omega_{\text {tail }}$ is the ratio of body and tail rotation rates, and $B_{o} / R_{b o d y}$ is the ratio of helical wave amplitude to the radius of the cylindrical body of the swimmer. The disagreement between experimental and computational velocity ratio results in Figures 7a,e is due to the weight of the helical tail resulting in extra translational friction on the channel walls [13, 14]. As the wave length increases, the agreement between velocity ratios improves dramatically and the novel set produces comparable or better results (see Figures 7b-d) except for large wave amplitudes where wall friction becomes important.

Likewise, the agreement on the ratio of body rotation rate to the tail rotation rate improves by increasing wave length and results. Furthermore the new coefficient set predicts the sensitivity of the rotation rate ratios to the wave amplitude successfully (see Figures $7 \mathrm{f}-\mathrm{h}$ ).

\section{Conclusions}

In this study, a parameterized experimental study is carried out to study the effect of helical wave geometry on a bio-inspired robot swimming in a cylindrical channel with both ends closed. The channel is filled with high viscosity Si-oil to satisfy the condition $\mathrm{Re}<0.1$. A total of 80 experiments are conducted and the velocity values are obtained by averaging out the observed displacements and rotations per unit time.

A series of CFD simulations are carried out to be able to implement a novel resistive force coefficient set for helical tails moving near cylindrical walls. Each 

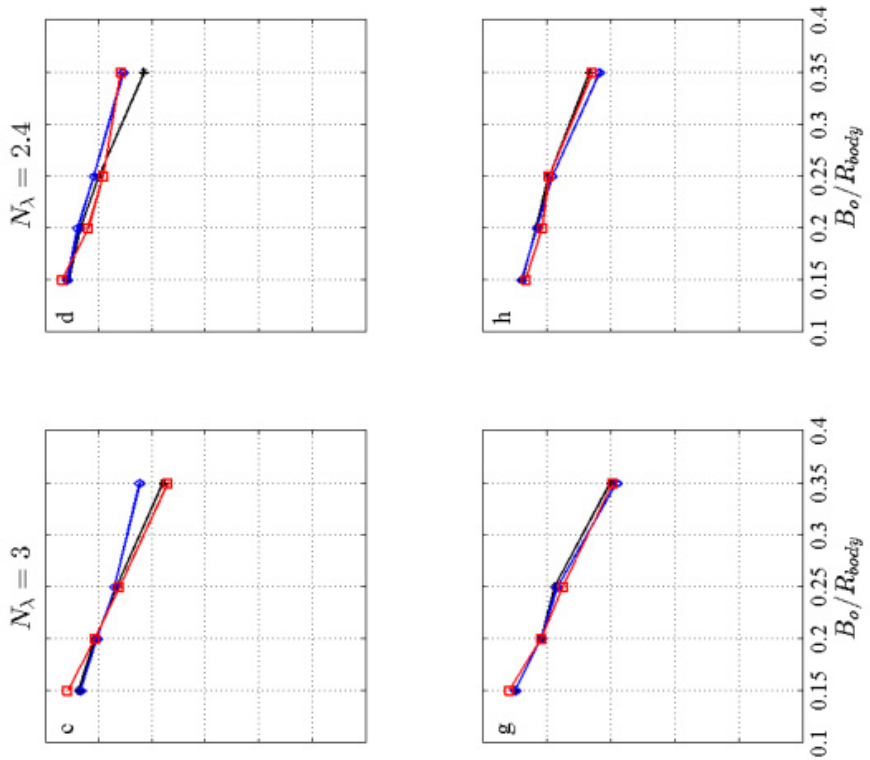

0
0
0
0
0
0
0
0
0
0
0
0
0
0
0
0
0
0
0
0
0
0
0
0
0
0
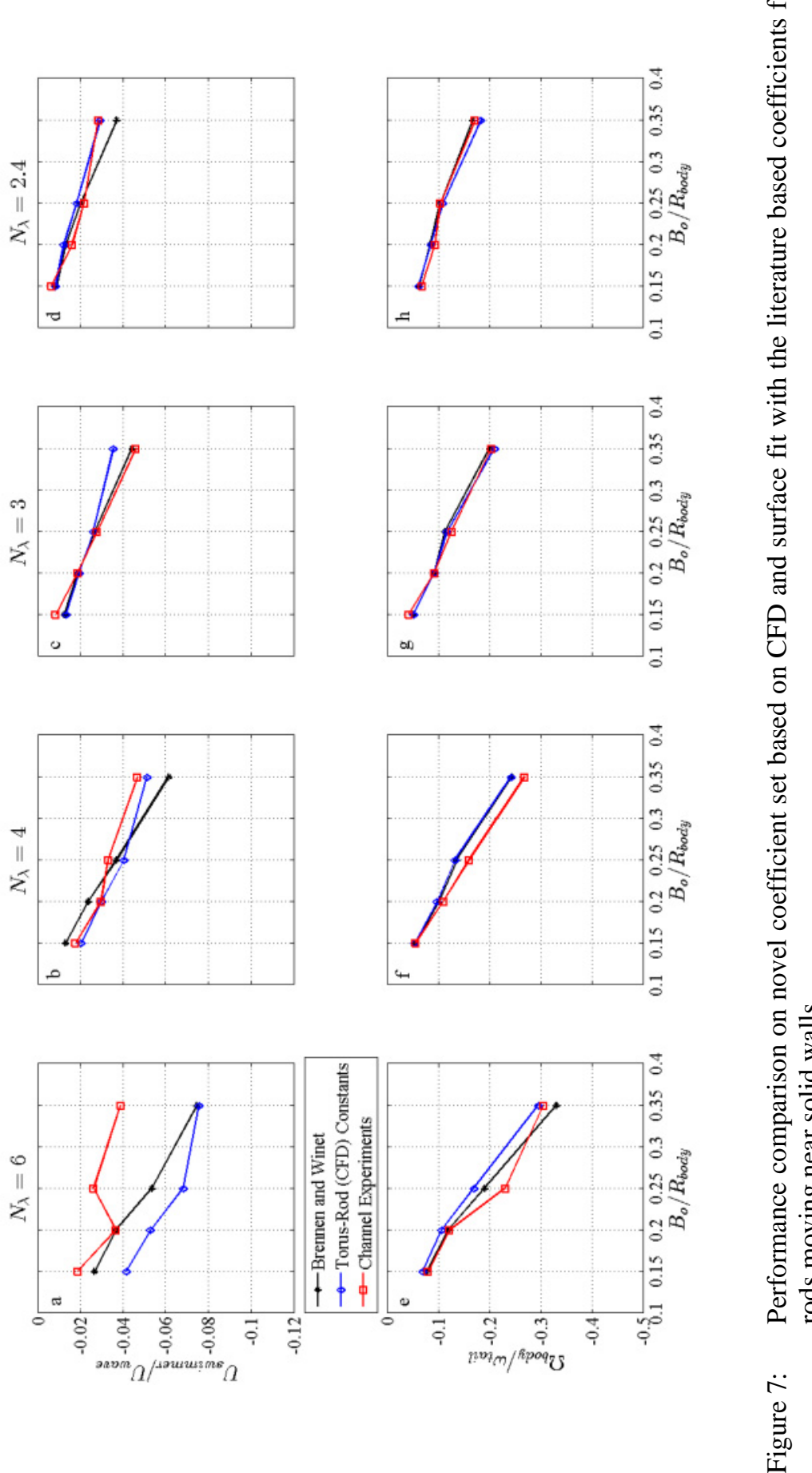
helical wave is represented by a separate full slender torus and a separate rod. The torus is replaced in a long channel to exclude the entrance effects whereas the rod is modeled such that the end effects are completely eliminated. A series of CFD simulations, which are governed by stationary incompressible Stokes equations, are carried out with parameterized wave amplitude, hence the proximity, and tail radius of concentric helical tails.

The fluid resistance values exerted on torus and rod are used in surface fit study, which utilized least square method with 95\% confidence interval, to determine the parameters of the novel resistive coefficient set. It is noted that the postulated coefficient set is established via a procedure of trial and error. The novel coefficient set includes corrections for $\mathrm{d} / r_{\text {tail }}, R_{c h} / r_{\text {tail }}$ and $\mathrm{d} / R_{c h}$. However, future study is required to include the wave length effect in the coefficient set.

The reduced-order model based on resistive force coefficients is utilized to predict the observed body rotation rates and forward swimming velocities given that the observed tail rotation rates are used as inputs, i.e. actuation frequency of the tail. The performance of the new set is compared against a coefficient set presented for rods moving plane boundaries. The latter set was successfully used to predict the velocity values of the bio-inspired robot previously.

For high wave numbers, i.e. small wave lengths, the reduced-order model fails to predict the swimming velocities; however, as the wave length increases the agreement improves dramatically to the point where the new set produces comparable, and in some cases superior with an error smaller than $1 \%$, results. However, the agreement deteriorates for the smallest and the largest wave amplitudes with $N_{\lambda}=3$. Overall, the new set predicts the effect of wave length and wave amplitude on the swimming velocities reasonably well.

\section{References}

[1] S. Martel, "Collective Methods of Propulsion and Steering for Untethered Microscale Nanorobots Navigating in the Human Vascular Network," Journal of Mech. Eng. Science, pp. 1505-1513, 2010.

[2] B. J. Nelson, I. K. Kaliakatsos, and J. J. Abbott, "Microrobots for Minimally Invasive Medicine,” Annu. Rev. Biomed. Eng., Vol. 12, pp. 5585, April, 2010.

[3] R. Bogue, "The Development of Medical Microrobots: A Review of Progress," Industrial Robot: An International Journal, Vol. 35, No. 4, pp. 294-299, 2008.

[4] J. Edd, S. Payen, B. Rubinsky, M. L. Stoller, and M. Sitti, "Biomimetic Propulsion for a Swimming Surgical Micro-Robot,” IEEE/RSJ Intelligent Robotics and Systems Conference (IROS), Vol. 3, pp. 2583-2588, Las Vegas, USA, October, 2003.

[5] L. Zhang, J. J. Abbott, L. Dong, B. E. Kratochvil, D. Bell, and B. J. Nelson, "Artificial Bacterial Flagella: Fabrication and Magnetic Control," Applied Physics Letters, Vol. 94, pp. 064107-1-3, February, 2009. 
[6] E. Shechter, S. Martel, "Principle of Motion Control of Bacterial MicroRobots Using Oxygen Gradients,” IEEE/ASME International Conference of Advanced Intelligent Mechatronics, Montreal, Canada, July 2010.

[7] J. Gray, G. J. Hancock, "The Propulsion of Sea-Urchin Spermatozoa," J. Exp. Biol., Vol. 32, pp. 802-814, 1955.

[8] J. Lighthill, "Flagellar hydrodynamics: The John von Neumann lecture," SIAM Rev., Vol. 18, pp. 161-230, 1975.

[9] C. Brennen, H. Winet, "Fluid Mechanics of Propulsion by Cilia and Flagella,” Ann. Rev. Fluid Mech., Vol. 9, pp. 339-398, 1977.

[10] E. Lauga, W. R. DiLuzio, G. M. Whitesides, H. A. Stone, "Swimming in Circles: Motion of Bacteria Near Solid Boundaries,” Biophysical Journal, Vol. 90, pp. 400-412, 2006.

[11] B. U. Felderhof, "Swimming at Low Reynolds Number of a Cylindrical Body in a Circular Tube,” Physics of Fluids, Vol. 22, 113604, 2010.

[12] N. Liron, R. Shahar, "Stokes Flow due to a Stokeslet in a Pipe," Journal of Fluid Mechanics, Vol. 86, Part 4, pp. 727-744, 1978.

[13] A. F. Tabak, S. Yesilyurt, "Experiments on In-channel Swimming of an Untethered Biomimetic Robot with Different Helical Tails,” In: The $4^{\text {th }}$ IEEE RAS/EMBS International Conference on Biomedical Robotics and Biomechatronics, Rome, Italy, 24-27 June, 2012.

[14] A. F. Tabak, S. Yesilyurt, "Experiment-Based Kinematic Validation of Numeric Modeling and Control of an Untethered Biomimetic Microrobot in Channel," The 12th IEEE International Workshop on Advanced Motion Control (AMC2012), Sarajevo, Bosnia and Herzegovina, March, 2012.

[15] A. F. Tabak, "Computational and Microhydrodynamic Modeling and Experiments with Bio-inspired swimming robots in cylindrical channels," PhD dissertation submitted to Faculty of Engineering and Natural Sciences, Sabanci University, Istanbul, 2012.

[16] E. M. Purcell, "Life at Low Reynolds Number," American Journal of Physics, Vol. 45, No. 1, pp. 3-11, 1977.

[17] E. Lauga, "Life Around the Scallop Theorem," Soft Matter., Vol. 72, pp. 19-54, 2010.

[18] Hancock, G.J., "The self-propulsion of microscopic organisms through liquids”, Proceedings of the Royal Society of London, Series A, Vol. 217, No. 1128, pp. 96-121, 1953.

[19] A. J. Hanson, H. Ma, "Visualizing Flow with Quaternion Frames,” Proc. of the conference on Visualization '94, Washington, D.C., pp. 108-115, 1994.

[20] J. Happel, H. Brenner, Low Reynolds Number Hydrodynamics, N.J.: Prentice-Hall, Inc., 1965.

[21] R. E. Johnson, "Hydromechanics of low-Reynolds-number flow. Part 5. Motion of a slender torus," Journal of Fluid Mechanics, Vol. 95, Part 2, pp. 263-277, 1979.

[22] O. Samson, "Low Reynolds number swimming in complex environments," $\mathrm{PhD}$ dissertation submitted to Department of Mathematics, Imperial College, London, 2010. 
[23] Y. Or, S. Zhang, R.M. Murray, "Dynamics and stability of low-Reynoldsnumber swimming near a wall" Siam Journal of Applied Dynamical Systems, Vol. 10, No. 3, pp. 1013-1041, 2011.

[24] L.D. Landau, E.M. Lifshitz, Fluid Mechanics Second Edition, Elsevier Butterworth-Heinemann, China, 2005.

[25] COMSOL, A.B., COMSOL Multiphysics User Guide, 2012.

[26] O. Schenk, K. Gärtner, "Solving unsymmetric sparse systems of linear equations with PARDISO,” Future Generation Computer Systems, Vol. 20, No. 3, pp. 475-487, 2004.

[27] L. F. Shampire, M. K. Gordon, Computer Solution of Ordinary Differential Equations: The Initial Value Problem, USA: Oxford University Press, 1985. 